\title{
Identifying new liver $X$ receptor alpha modulators and distinguishing between agonists and antagonists by crystal ligand pocket screening
}

\author{
Jia-Hau Lee ${ }^{\ddagger 1,2}$, Chien-Fu Huang ${ }^{\ddagger 3}$, Yi-Jing Chuang ${ }^{1}$, Chang-Yin Lee ${ }^{4,5}$, Wen-Hao Yu ${ }^{1}$, \\ Chin-Chung $\mathrm{Wu}^{2,6}$ \& Ying-Ting Lin*,1,6 (iD) \\ ${ }^{1}$ Department of Biotechnology, College of Life Science, Kaohsiung Medical University, Kaohsiung, Taiwan \\ ${ }^{2}$ Graduate Institute of Natural Products, College of Pharmacy, Kaohsiung Medical University, Kaohsiung, Taiwan \\ ${ }^{3}$ Department of Biological Science \& Technology, I-Shou University, Kaohsiung, Taiwan \\ ${ }^{4}$ Department of Chinese Medicine, E-DA Cancer Hospital, Kaohsiung, Taiwan \\ ${ }^{5}$ College of Medicine, The School of Chinese Medicine for Post Baccalaureate, I-Shou University, Kaohsiung, Taiwan \\ ${ }^{6}$ Drug Development \& Value Creation Research Center, Kaohsiung Medical University, Kaohsiung, Taiwan \\ *Author for correspondence: Tel.: +886 7322 1514; Fax: +886 7312 5339; ytlin@kmu.edu.tw \\ ${ }^{\ddagger}$ Authors contributed equally
}

Background: Modulators of LXR $\alpha$ are of high pharmacological interest as LXR $\alpha$ regulates fatty acid metabolism, inflammatory processes and cancer. We aim to identify new LXR $\alpha$ modulators and to recognize a distinguishable feature of agonists. Results \& methodology: The ligand self-dock and largestcavity-size searching purposely located two appropriate ligand-binding sites to reach the two aims. One is identifying the new modulators from Maybridge library. 20 new compounds are confirmed by the in vitro reporter gene assay. The other is denoting an agonist by at least one best docking pose having one hydrogen bond to LXR $\alpha$ Helix12 His421. Conclusion: Based on the quality $x$-ray binding pocket, we can identify new $\mathrm{LXR} \alpha$ modulators and distinguish between agonists and antagonists by molecular docking.

\section{Graphical abstract:}

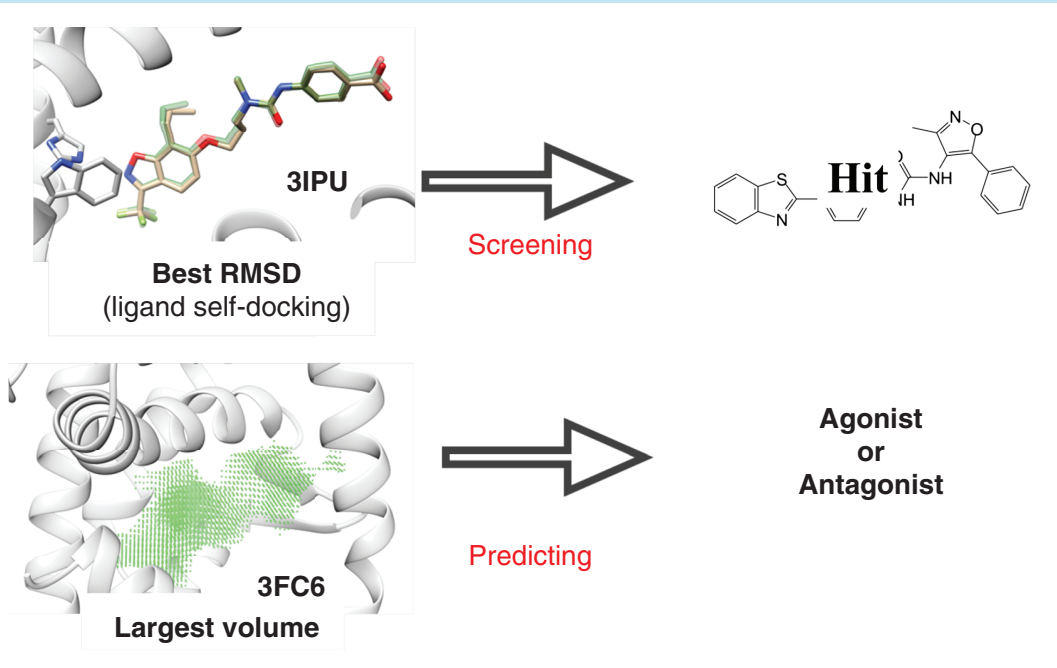

First draft submitted: 10 March 2020; Accepted for publication: 14 April 2020; Published online: 
Keywords: agonist $\bullet$ antagonist $\bullet \mathrm{LXR} \alpha \bullet$ molecular docking $\bullet$ self-docking $\bullet$ virtual screening $\bullet \mathrm{x}$-ray ligand-binding sites

Liver X receptor alpha $(\mathrm{LXR} \alpha)$ is one of the LXR subtypes belonging to the nuclear receptor superfamily. As a ligand-activated transcription factor, $\mathrm{LXR} \alpha$ contains a highly conversed DNA-binding domain and ligand-binding domain (LBD), regulating the expression of genes through binding to the region of the so-called LXR response element of the downstream genes. LXR $\alpha$ participates in the regulation of lipid metabolism and inflammation, and also cancer. Nonalcoholic fatty liver disease is a common liver disease associated with LXR $\alpha$ [1,2]. LXR $\alpha$ also showed oncogenic properties in cancer cells, such as gastric and ovarian cancer [3]. Thus, LXR $\alpha$ can be an essential pharmaceutical target to alter the biological process for different diseases [4]. LXR $\alpha$ modulators, by their transcriptional activities, can be divided into agonists or antagonists. Depending on different expressions or organ heterogenetic of $\operatorname{LXR} \alpha$, the agonist in principle can relieve nonalcoholic fatty liver disease symptoms and the antagonist can be used to treat cancer. $\operatorname{LXR} \alpha$ is an attractive target for its potential functions, and new LXR $\alpha$ modulators have a chance to be developed into new drugs [5,6]. To meet pharmaceutical demands, we need to identify more LXR $\alpha$ modulators and also to distinguish between the modulator types, especially for the early phase of drug discovery [7]. Here, we selected the appropriate crystal pockets as two binding site models for efficient molecular-docking screening to identify novel $\mathrm{LXR} \alpha$ modulators and differentiate types of modulators.

To identify new LXR $\alpha$ modulators, we chose a suitable binding site model to conduct efficient structurebased molecular-docking screening. The molecular dockability $[8,9]$ of the $\mathrm{x}$-ray crystal ligand-binding pocket was examined by the self-docking of crystal complex ligands. If the ligand after self-docking can correctly find its crystal pose, it can indicate the dual facts that the native binding pocket and the methodology of molecular docking used are both reliable. The similarity between the best docking pose and experimental crystal pose is calculated using the root-mean-square deviation (RMSD) [10]. In primary molecular-docking screening, we also utilized two restrictions to reduce the number of purchased library compounds: consensus scoring [11] and Lipinski's rule [12]. The details of the methodology and procedure for efficient virtual screening are described in the Methods section. We screened 25 candidates and further analyzed them by $\operatorname{LXR} \alpha$ transactivation luciferase assay [13]. 20 hits were classified as significantly either enhancing or reducing transactivation.

To differentiate types of modulators, we needed to choose another suitable binding site model to distinguish the agonism of the hits by structure-based molecular docking screening. According to the standard theory of nuclear receptor transactivation mechanisms, an agonist binding in the core of the LBD with contact to the AF2 function site induces a conformational change which can introduce a binding site for coactivators in a groove formed majorly by Helix12, leading to effective transactivation [14-16]. Molecular docking to see whether a hit can dock into the LBD and whether the hit can also show contact with LXR $\alpha$ Helix12 His421, which may denote its agonism. If not, lack of contact with His421 indicates antagonism. For this simulation, we used the largest binding pocket, which can accommodate the greatest variety of LXR $\alpha$ ligand structures. We found that the x-ray LXR $\alpha$ complex (Protein Data Bank [PDB] ID: 3FC6) has the largest ligand-binding pocket. The methods used to decipher the agonism of hits are described below. The docking poses of a hit tending to have the hydrogen bond binding to LXR $\alpha$ Helix12 His421 strongly imply that the compound is an agonist, while lack of binding implies an antagonist. The best agonist hit and the best antagonist hit in this study were demonstrated to qualitatively illustrate such features.

Appropriate crystal-binding pockets as models of ligand-binding sites are crucial for the success of moleculardocking simulations. Each binding cavity of the x-ray crystal LXR $\alpha$ complex can reflect a snapshot of a true dynamic ligand-binding pocket. Instead of computationally altering the pocket residues, we kept it frozen with the two selected $\mathrm{x}$-ray coordinates of the ligand-binding environment for hit screening and to distinguish agonism. These two approaches may provide more efficient and more correct results for structure-based molecular modeling for both purposes.

\section{Computational \& experimental methods Molecular docking}

LigandFit [17] is a molecular docking method for accurately docking ligands into protein-binding sites and also for high-throughput hit screening. All molecular docking experiments using Discovery Studio 2.5 [18] were performed on an Asus computer with an Intel Core i7 $4.00 \mathrm{GHz}$ processor, running Windows 7. The energy grids of set binding sites can be further calculated by three different force fields, Dreiding [19], consistent force field (CFF) [20] and PLP1 [18] as implemented in LigandFit. Before molecular docking, the ligand is removed from the active site, 
leaving a binding site model for molecular simulation. We added hydrogen atoms to LXR $\alpha$ receptors and their ligands according to the default values of the CHARMM force field [21]. DockScore [17] was then used to estimate the interaction energy between calculated ligand poses and the LXR $\alpha$ ligand-binding pocket. We used the same methodology and molecular docking procedure as a common base to inspect the quality of x-ray crystal-binding pockets [9]. Ligand self-docking is an important metric for evaluating docking methods [22,23] by correctly simulating the crystal ligand pose. We use self-docking to examine the intrinsic quality of $\mathrm{x}$-ray binding sites since our docking method and procedure were the same. We emphasize the validation of the resulting RMSD for measuring the accuracy of the docking methodology and the quality of the $\mathrm{x}$-ray ligand pocket. Ligand self-docking has the same procedure as mentioned [9].

\section{Utility-based selection of $\mathrm{x}$-ray binding sites}

Binding site for molecular docking was defined by the occupied space of the cocrystal ligand, which was set by expanding the ligand hydrogen radius to $2.0 \AA$ and heavy ligand atom to $2.5 \AA$ (default settings) until reaching residues. We collected $19 \mathrm{x}$-ray crystal protein chains of human $\mathrm{LXR} \alpha$ cocomplexed with ligands [14,24-29] deposited in the PDB (more than one decade) [30]. The same definition can also calculate the size of each binding site. The best $\mathrm{x}$-ray binding site for new modulator screening was examined by the self-docking strategy, while the $\mathrm{x}$-ray binding site for distinguishing agonism used the largest one.

\section{New modulator screening \& consensus scoring}

To be as close to reality as possible, we set all the crystal protein residues as still. All crystal waters were also removed since the orientation of water is unknown. A maximum of ten poses was set to be saved after molecular docking. Then, for each docking pose, 11 scoring functions, LigScore1 [17], LigScore2 [17], DockScore [17], PMF [31], PMF04 [32], Ludi1 [33,34], Ludi2 [33,34], Ludi3 [35], PLP1 [18,36], PLP2 [18,36] and Jain [37], were used for consensus scoring. Consensus scoring was applied as follows: each library compound can have up to ten after-dock poses, and each pose has 11 scores. If a pose's score in one of the 11 scoring functions was within the top $6 \%$ of total library docking poses, by definition, the pose gained one consensus point. An in-house Ruby (www.ruby-lang.org) programming script for docking-pose-based consensus scoring was used to calculate consensus points.

\section{Lipinski's rule of five}

In drug discovery, desired molecules can fulfill particular criteria for further drug development. Lipinski's rule of five (ROV) [12] is a traditional way to address the drug-likeness of the organic compounds. ROV rules include no more than ten hydrogen bond acceptors, no more than five hydrogen bond donors, molecular weight (mass) lower than $500 \mathrm{Da}$ and partition coefficient (AlogP) smaller than five. As improvements for the drug-likeness of organic compounds, Veber and coworkers [38] showed that the polar surface area (PSA) [39] and the number of rotatable bonds (RBs) can be discriminating molecular descriptors. Two additional criteria can be PSA $<140 \AA^{2}$ and RBs $\leq 10$. Those compounds fulfilling Veber's rules are expected to have good oral availability. To further lower time and cost, we wanted to identify the best hits successfully passing ROV and the two additional criteria. The related molecular properties were calculated by the Discovery Studio 2.5 module for molecular properties.

\section{Compound library}

The Maybridge Screening collection, with $>57,000$ compounds, was the compound library used for screening. All Maybridge compounds were optimized within Pybel [40,41] using the MMFF94 forcefield until the structure's energy reached the RMSD gradient of $0.05 \mathrm{kcal} / \mathrm{mol} / \AA$ [42]. The partial charges and the optimized structures of chemical compounds were automatically set and saved in mol file format for further hit screening of molecular docking.

\section{$\mathrm{LXR} \alpha$ transactivation luciferase assay}

LXR $\alpha$ transactivation tests including Huh7 cells were used for the luciferase assay. Transfections were done in Huh7 cells using Lipofectamine Plus (Life Technologies, Inc., MD, USA) according to the manufacturer's instructions. In each transactivation assay, the following plasmids were involved: a reporter containing five Gal4 binding sites $(0.2 \mu \mathrm{g})$, an expression plasmid for LXR $\alpha$ LBD fusion protein $(0.6 \mu \mathrm{g})$ and $60 \mathrm{ng}$ SV 40-Relina plasmid. Pure compounds were added $12 \mathrm{~h}$ after cell transfection was done for 1 day. Cells were collected and lysed for luciferase assays following the manufacturer's instructions. L783483, being a pan-agonist for the nuclear receptors, was used 
Table 1. List of 19 binding sites and root-mean-square deviation values of docked poses and crystal poses.

\begin{tabular}{|c|c|c|c|c|c|}
\hline \multirow[t]{2}{*}{ PDB ID (chain) } & \multirow[t]{2}{*}{ Resolution (Å) } & \multirow[t]{2}{*}{ Binding site size $\left(\AA^{3}\right)$} & \multicolumn{3}{|c|}{ RMSD (Å) } \\
\hline & & & Dreiding & CFF & PLP1 \\
\hline $1 \mathrm{UHL}(\mathrm{B})$ & 2.90 & 416.75 & 0.92 & 1.02 & 2.73 \\
\hline $2 A C L(B)$ & 2.80 & 368.75 & 0.73 & 0.78 & 0.79 \\
\hline $2 \mathrm{ACL}(\mathrm{D})$ & 2.80 & 336.38 & 0.82 & 0.87 & 0.69 \\
\hline $2 A C L(F)$ & 2.80 & 376.50 & 0.84 & 0.71 & 1.18 \\
\hline $2 \mathrm{ACL}(\mathrm{H})$ & 2.80 & 373.25 & 0.59 & 0.60 & 0.59 \\
\hline 3FAL (B) & 2.36 & 369.38 & 0.82 & 2.55 & 2.07 \\
\hline 3FAL (D) & 2.36 & 393.13 & 2.57 & 2.57 & 2.05 \\
\hline $3 F C 6$ (B) & 2.06 & 472.50 & 0.65 & 0.72 & 0.76 \\
\hline 3FC6 (D) & 2.06 & 473.88 & 0.50 & 0.57 & 0.66 \\
\hline 3IPQ (A) & 2.00 & 451.63 & 0.46 & 0.48 & 0.58 \\
\hline 3IPS (A) & 2.26 & 432.50 & 0.54 & 1.09 & 0.94 \\
\hline 3IPS (B) & 2.26 & 434.38 & 1.73 & 1.65 & 10.34 \\
\hline 3IPU (A) & 2.40 & 443.63 & 0.37 & 0.40 & 0.59 \\
\hline 3IPU (B) & 2.40 & 436.75 & 0.37 & 0.46 & 12.66 \\
\hline $5 \mathrm{AVI}(\mathrm{A})$ & 2.70 & 372.38 & 0.47 & 0.46 & 0.49 \\
\hline $5 A V I(C)$ & 2.70 & 375.00 & 0.47 & 0.47 & 0.47 \\
\hline $5 A V L(A)$ & 2.80 & 401.25 & 1.09 & 0.69 & 1.22 \\
\hline 5 HJS (A) & 1.72 & 421.00 & 0.55 & 0.63 & 1.55 \\
\hline $5 \mathrm{HJS}$ (B) & 1.72 & 422.38 & 0.80 & 0.83 & 0.74 \\
\hline Average & 2.42 & 409.02 & 0.80 & 0.92 & 2.16 \\
\hline
\end{tabular}

as an agonist standard. The LXR $\alpha$ ligand transactivation activities of 25 screened Maybridge compounds were tested with $10 \mu \mathrm{M}$ as the primary screening assay.

\section{Results \& discussions}

Best $x$-ray binding site for molecular docking screening

The quality of the binding sites was our first concern for highly accurate molecular docking virtual screening for LXR $\alpha$. X-ray crystallography can resolve high-resolution structures at the atomic level and structural details, but the intrinsic resolution of x-ray crystallography is still not enough for accurate structure-based drug design. Theoretically, a $2 \AA$ deviation from reality at a critical binding-site residue can lead to failures in drug design, since the length of a single bond is $<2 \AA$. We can see that the average resolution of the collected LXR $\alpha$ PDB complexes is $2.42 \AA$ (Table 1, resolution column) [14,24-29]. Such a departure from reality, if it happens in the binding site, can severely decrease the accuracy of molecular docking, and thus, the efficiency of virtual screening. Therefore, a further inspection of the 'dockable nature' $[8,9]$ of the ligand pockets of each crystal complex is essential for molecular docking. To raise our confidence for the crystal-binding pockets of LXR $\alpha$ for molecular docking, we used an additional strategy, self-docking, in which a geometry-randomized crystal ligand docks back into the natural crystal-binding cavity. The degree of regaining the ligand-bound pose is initially one of the central criteria for the correctness of the docking method [43,44], but here we used it to check the reliability and suitability of crystal-binding sites for hit screening. The shape overlap between the $\mathrm{x}$-ray pose and the best ligand-docking pose was calculated by RMSD. The 19 results of self-docking are listed by the energy grids of the three different force fields, Dreiding, CFF and PLP1, in Table 1, where the RMSD ranged from 0.37 to $2.57 \AA$, from 0.40 to $2.57 \AA$ and from 0.47 to $12.66 \AA$, respectively, as shown in the three violin distribution graphs (Figure $1 \mathrm{~A}$ ). We can see that better crystal protein resolution did not ensure lower RMSD values after self-docking. The best docking pose of the 3IPU ligand (Dreiding) was similar to the native bound crystal pose, with an RMSD of $0.37 \AA$ (Figure 1B). Thus, the 3IPU ligand-binding site was considered to be the best $\mathrm{x}$-ray binding site for the new molecular docking modulator screening of LXR $\alpha$. Lastly, we stress here that all the protein residue side chains were not allowed to move to remain close to reality as possible, and also that crystal waters were also removed since the orientation of 
(A)

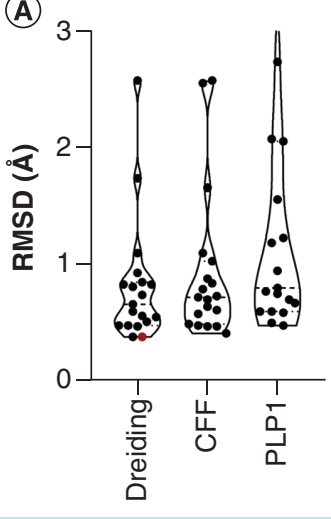

(B)

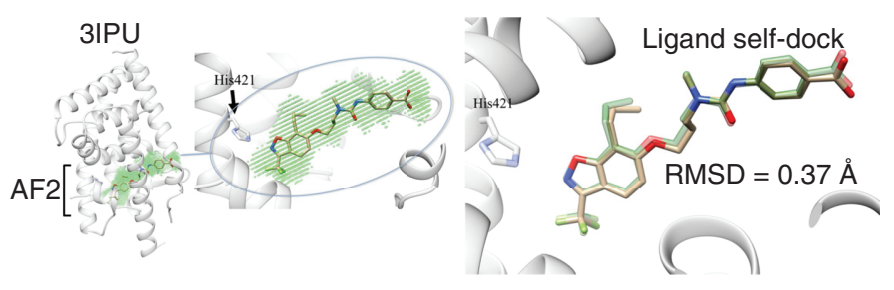

Figure 1. Ligand self-docking. (A) The three violin distribution graphs show that all RMSD values of 19 liver $X$ receptor alpha ligands ranged from 0.37 to $2.57 \AA$, from 0.40 to $2.57 \AA$ and from 0.47 to $12.66 \AA$, on the energy grids of three different force fields, Dreiding, CFF and PLP1, respectively. All docked poses were compared spatially with their native crystal poses after the ligand self-docking. The smallest one is represented by the dark red spot. (B) The best spatial RMSD, $0.37 \AA$, occurring in the 3IPU crystal ligand-binding pocket, illustrated with zoom-out, middle and zoom-in pictures. The critical residue, His421, is shown.

CFF: Consistent force field; RMSD: Root-mean-square-deviation.

water is unknown in $\mathrm{x}$-ray crystallography. In this case, there is no water existing in this binding pocket. We believe that each $\mathrm{x}$-ray binding site reflects a snapshot of dynamic ligand-pocket interactions. Thus, we left the selected $\mathrm{x}$-ray coordinates unchanged, rather than computationally altering the ligand-binding surroundings of the pocket. So, in this study, we excluded the method of molecular dynamics.

\section{Possible LXR $\alpha$ modulators by screening \& consensus scoring}

The best 25 hit compounds were purchased for in vitro reporter gene assays. The chemical structures are shown in Figure 2. Each hit was picked out by the following sequence: when the best $\mathrm{x}$-ray binding site was located, we did molecular docking to each of $>57,000$ library compounds. This step generated about 167,000 docking poses with 11 scoring functions, of which 228 screening compounds had consensus points $>7$. Ligand-receptor binding may involve a variety of mathematical forms for noncovalent interactions, presenting differently in diverse ligand-receptor interactions [45-47]. As the literature shows [22,23], no single scoring function can handle all types of binding pockets equally well. To ensure finding the best hit, we did consensus scoring [45-47] for all docking poses. If the specific score for docking poses was within the top $6 \%$ of total docking poses it gained one consensus point. We took all docking poses as the total population rather than only the best docking poses from the library compound, and thus, the consensus scoring was based on docking poses.

Moreover, the selected compounds have different structures than the parent crystal ligand. Scaffold hopping [48] seemed to be easily achieved in this molecular docking screening. Note that we chose hits by the prediction of ligand-receptor interaction for our concept validation. However, to reduce the time and cost of the luciferase assay, we further considered the ROV, PSA and number of RBs for the possible drug development of these hits. The related ROV values are listed in Supplemental Table 1. At last, we purchased 25 compounds for the in vitro reporter gene assay.

\section{Novel LXR $\alpha$ modulators by in vitro reporter gene assay}

The best 25 hit compounds were evaluated by in vitro LXR $\alpha$ luciferase reporter gene assay. The luciferase activities for all hit structures are shown in Figure 3. Luciferase assays are a typical cell-based experimental method used to measure the transactivation activity of LXR $\alpha$. The 25 hits and L783483 [49] were assayed in transfected Huh7 cells at $10 \mu \mathrm{M}$. The control was the normal luciferase activity of the system when no compound was added. L783483 is a pan-agonist for nuclear receptors, here used as an agonist standard for comparison. The outcome of luciferase chemiluminescence reveals the degree of LXR $\alpha$ transactivation, showing whether a hit activates or inhibits it. More than half of 20 hits show a significant influence on LXR $\alpha$ transactivation. Then we further classified the identified 


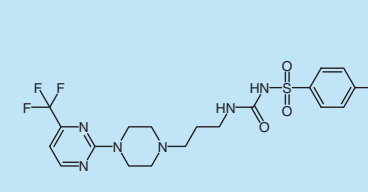

1

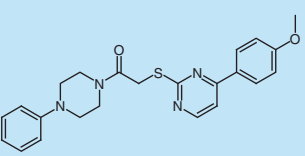

6

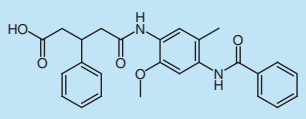

11

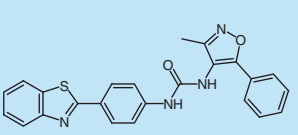

16

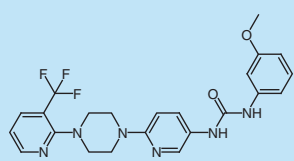

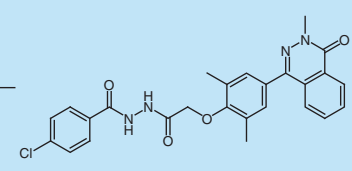

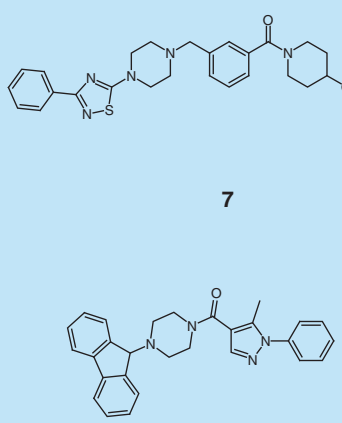

12

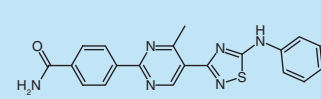

17

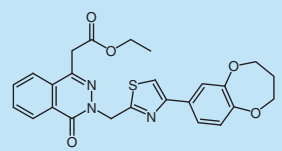

22

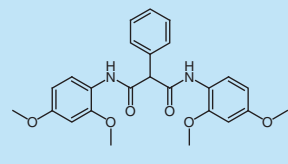

3

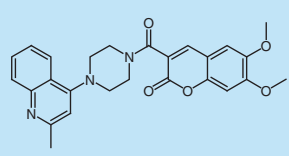

13
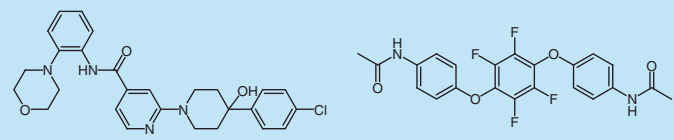

14

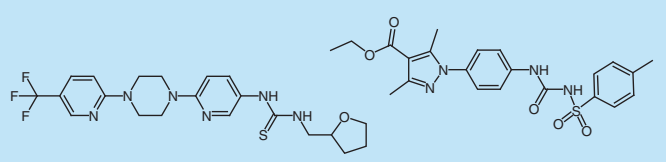

18

19

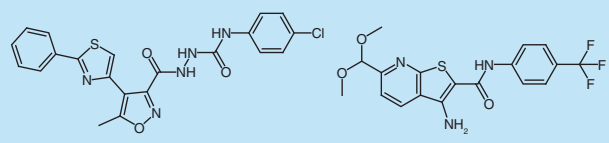

23

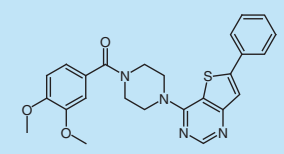

5

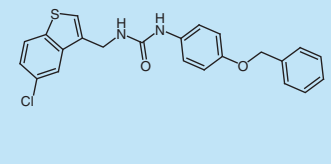

10

Figure 2. Chemical structures of compounds 1-25 were screened using the best selected 3IPU ligand-binding pocket. Significantly different structures other than the parent ligand are shown. Scaffold hopping is observed in these compounds. The values of Lipinski's rule of five, polar surface area and umber of rotatable bonds were also calculated and listed in Supplementary Table 1.

hits into five groups. In group 1 , six agonists $(\mathbf{1}, \mathbf{9}, \mathbf{1 1}, \mathbf{1 2}, 22$ and 24) showed higher LXR $\alpha$ transactivation activity of at least $20 \%$ more than control. Notably, among group 1 , the four agonists $(\mathbf{1}, \mathbf{9}, \mathbf{1 1}$ and $\mathbf{2 2})$ had LXR $\alpha$ transactivation higher than or similar to that of L783483. Group 2 had the 14 antagonists $(\mathbf{2 - 8 , 1 3 , 1 4 , 1 6 , 1 9}$, 20, 23 and 25), showing the LXR $\alpha$ transactivation activity less than $80 \%$ of blank control. We also noticed that two antagonists ( 2 and $\mathbf{5}$ ) showed significant antagonism less than $20 \%$ of control and most antagonists show less than $50 \%$ of control. Group 3 had five hits $(\mathbf{1 0}, \mathbf{1 5}, \mathbf{1 7}, 18$ and 21) showing no significant alteration of LXR $\alpha$ transactivation activity and were similar $(\leq \pm 20 \%)$ to blank control. Overall, $80 \%$ of our purchased LXR $\alpha$ modulators demonstrated molecular docking of selected $x$-ray binding sites, suggesting that this method could provide an extremely efficient way of virtual screening.

\section{Distinguishing agonism}

According to the recognized concepts of nuclear receptor transactivation mechanisms, a ligand is necessary to bind to the AF2 function site for the activation of a specific nuclear receptor [14-16]. In LXR $\alpha$ (as well as other nuclear receptors), we can also see that a full agonist always exhibits a feature whereby the head of the ligand structure body has a direct hydrogen bond link with the AF2 Helix 12 His 421 in x-ray crystallography images. We used this criterion to see if our molecular docking simulation could differentiate the agonism of a virtual screening hit. We 


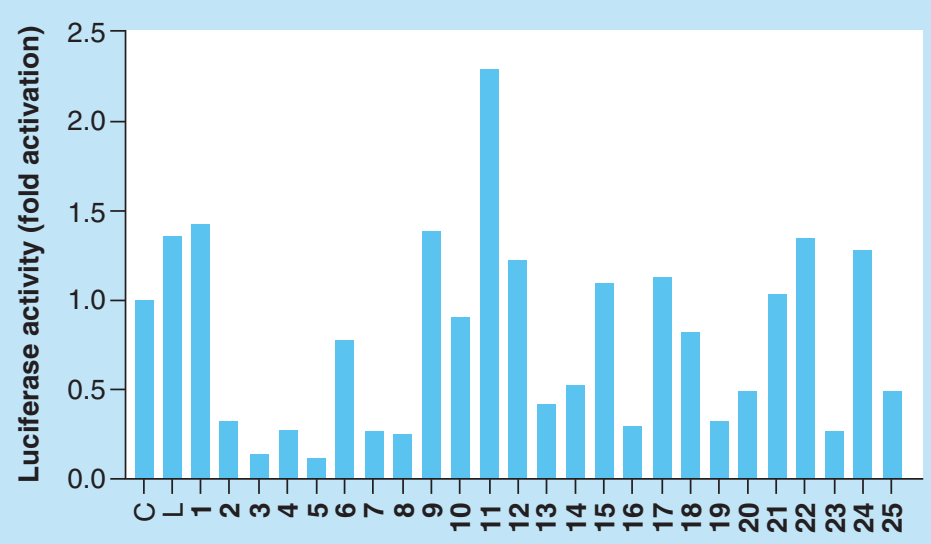

Figure 3. LXR $\alpha$ ligand transactivation activities of 25 screened compounds are shown as primary screening by luciferase assays.

C: Control was the normal luciferase activity of the system when no compound was added; L: L783483 is a pan-agonist for the nuclear receptors used as an agonist standard.

Figure 4. X-ray crystal ligand-binding sites. (A) A violin distribution graph showing all size values of 19 collected $x$-ray crystal ligand-binding sites ranging from 336.38 to $473.88 \AA^{3}$. The largest is represented by the dark red spot. (B) The biggest crystal ligand-binding site located on the 3FC6.D protein crystal has pocket size, $473.88 \AA^{3}$, illustrated by yellow dots, while the smallest binding site located on the 2ACL.D crystal is $336.38 \AA^{3}$ in size, represented by the blue dots.
(A)

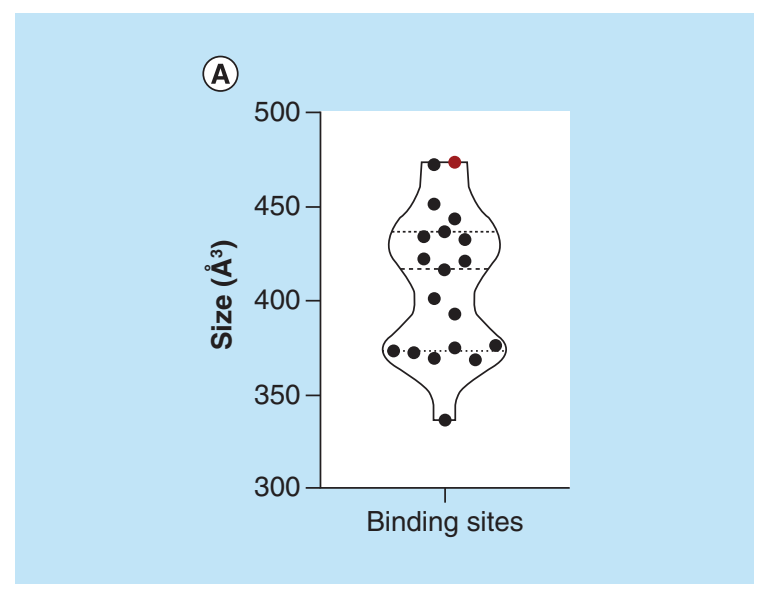

believe any computational alteration of the protein backbone structure may cause some amount of uncertainty. A specific binding site can be seen as a snapshot of the dynamic reality of $\operatorname{LXR} \alpha$ complex. We tried to avoid unnecessary modifications to the original structure coordinates revealed by $\mathrm{x}$-ray crystallography. To allow the maximum docking poses, we took the largest binding pocket, which may accommodate the greatest variety of LXR $\alpha$ ligand structures. The volumes of different binding sites, as well as the largest-size ligand-binding pocket (PDB ID: 3FC6), are listed in Table 1 (binding site columns) and illustrated in Figure 4A and B. Our aim was to see if hits could dock into the LBD and whether the hit furthered show a hydrogen bond link with 3FC6 LXR $\alpha$ AF2 His421 [14-16] or not. As a result of all simulation observations, the best antagonist (compound 5) and the best agonist (compound 11) in our study were taken as examples. The docking pose of compound $\mathbf{5}$ showed multiple definite binding interactions: a strong $\pi-\pi$ interaction with Trp443 (head of the ligand) and hydrogen bonds with Asn225 and Arg305 (tail of ligand) as shown in Figure 5A. The docking pose of compound 11 also demonstrated multiple definite binding interactions: strong $\pi$-cation interactions with Arg305 (tail of ligand) and hydrogen bonds with Ser264 (body of ligand) and, most importantly, with His421 (head of the ligand) as shown in Figure 5B. By observing plentiful docking poses of nuclear receptor ligands including LXR $\alpha$ ligands, we can safely draw a qualitative conclusion that either a potent binding nuclear receptor agonist or antagonist tends to have multiple significant interactions in the binding site and that the distinguishing feature is that the full agonist must have the hydrogen bond binding to Helix 12 His 421 . Furthermore, the particular structural features of the strong LXR $\alpha$ agonists are having strong hydrogen acceptors on the heads of agonists, which can fit spatially into 


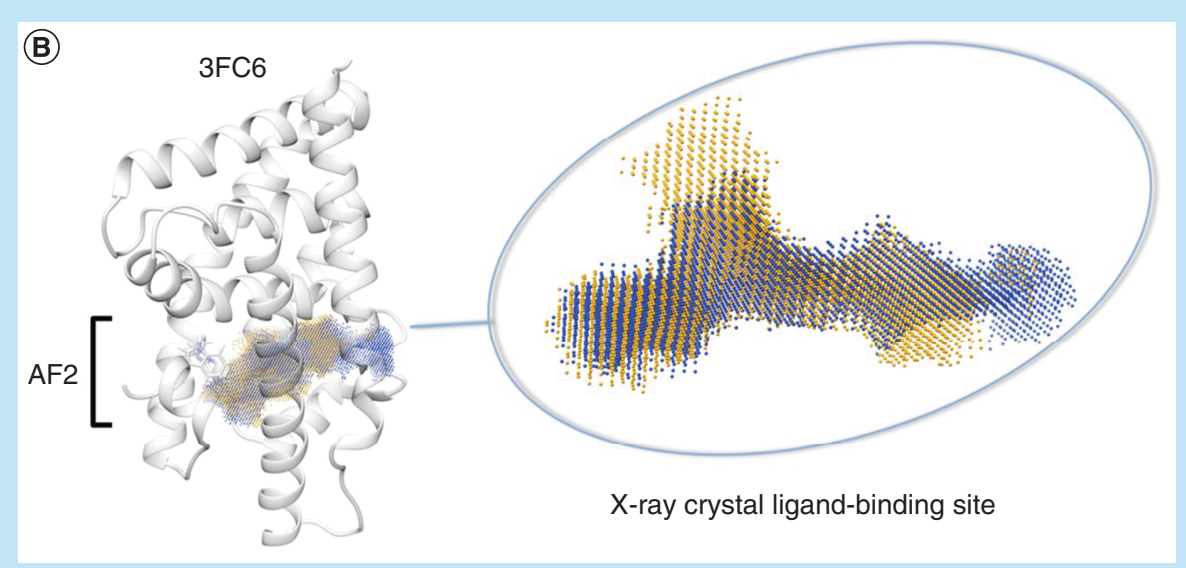

Figure 4. X-ray crystal ligand-binding sites (cont.). (A) A violin distribution graph showing all size values of 19 collected $x$-ray crystal ligand-binding sites ranging from 336.38 to $473.88 \AA^{3}$. The largest is represented by the dark red spot. (B) The biggest crystal ligand-binding site located on the 3FC6.D protein crystal has pocket size, $473.88 \AA^{3}$, illustrated by yellow dots, while the smallest binding site located on the $2 A C L . D$ crystal is $336.38 \AA^{3}$ in size, represented by the blue dots.
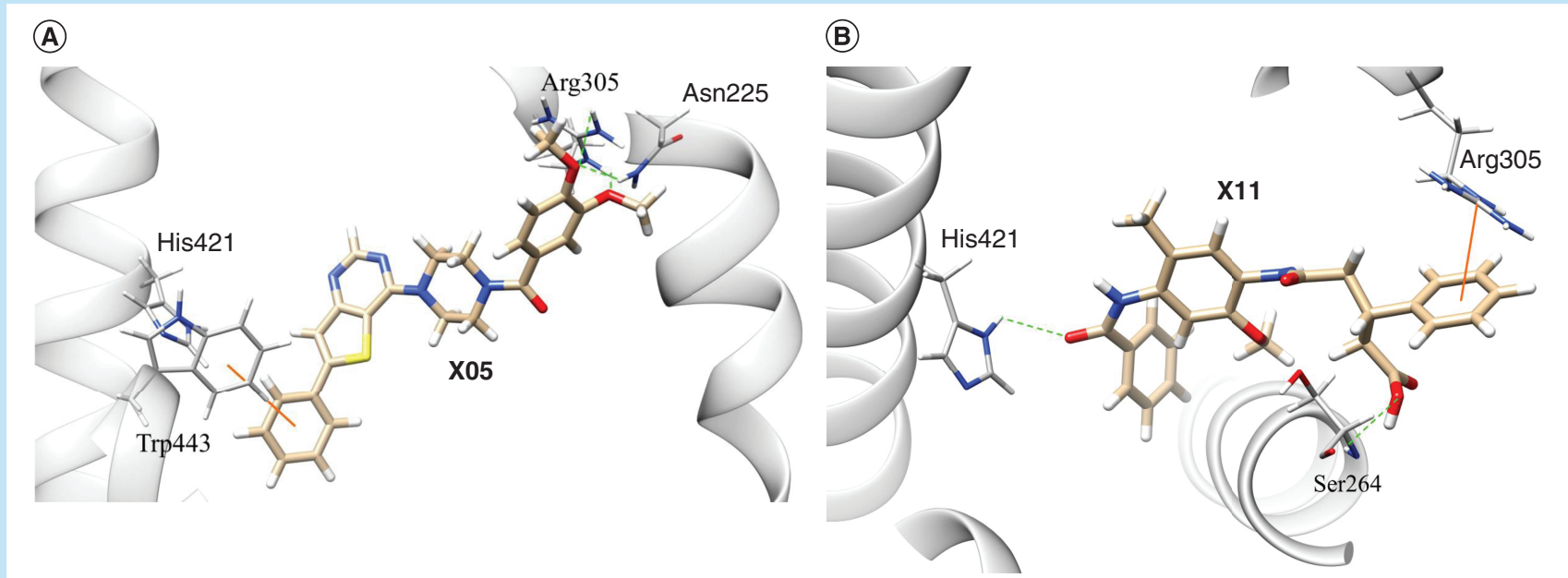

Figure 5. Analysis of the docking poses of compound 5 and 11. (A) Docking pose of compound 5 shows multiple definite binding interactions: a strong $\pi-\pi$ interaction with Trp443 (head of the ligand) and hydrogen bonds with Asn225 and Arg305 (tail of the ligand). (B) Docking pose of compound 11 also shows multiple definite binding interactions: strong $\pi$-cation interactions with Arg305 (tail of the ligand) and hydrogen bonds with Ser264 (body of the ligand) and, most importantly, a hydrogen bond with His421 (head of the ligand).

the Helix12 His421 vicinity surroundings. For example, the trifluoromethyl group in Y1, the carbonyl group in Y9 and Y22 and the carboxylic acid in Y11 serve as such hydrogen acceptors. In the molecular docking simulation, the greater frequency of this hydrogen bond link in hit docking poses indicates that the hit is a full agonist (a strong binder with Helix12 His421). In contrast, in a strong binding antagonist hit it is hard to see any such hydrogen bond link in any docking pose (a strong binder with no Helix12 His421 link).

\section{Conclusion}

Modulators of $\mathrm{LXR} \alpha$ are of high pharmacological interests as $\mathrm{LXR} \alpha$ regulates fatty acid metabolism, inflammatory processes and even related to cancer. Based on the suitably selected experimental native binding sites of $\mathrm{x}$-ray receptor complexes for molecular docking, we aim both to identify the new LXR $\alpha$ modulators and to recognize 
the distinguishable feature between agonists and antagonists. Our two approaches, ligand self-dock and largestcavity-size searching, purposely located two appropriate ligand-binding site models to reach the following two aims: for identifying new modulators, after sorting all the docking poses of a chemical library of 57,000 compounds, out of 25 purchased compounds, in vitro reporter gene assay confirmed up to 20 agonists or antagonists; and for distinguishing types of the modulators, the results of docking pose indicates a feature that at least one pose, out of ten poses, having one hydrogen bond to LXR $\alpha$ Helix 12 His 421 denotes its agonism. Thus, the main structural features for the LXR $\alpha$ agonists are having strong hydrogen acceptors on the heads of agonists fitting to the Helix 12 His421 vicinity surroundings. Overall, the utility-based selections of $\mathrm{x}$-ray binding sites showed that molecular docking not only can screen new active modulators but also can differentiate between agonism and antagonism.

\section{Future perspective}

In the future, new LXR $\alpha$ modulators will have a chance to be developed into new drugs. Appropriate crystal-binding pockets as models of ligand-binding sites are important for the success of molecular docking simulations. Each binding cavity of the $\mathrm{x}$-ray crystal $\mathrm{LXR} \alpha$ complex can reflect only a snapshot of a true dynamic ligand-binding pocket. The two selected $\mathrm{x}$-ray coordinates of the ligand-binding environment were used for hit screening and to distinguish agonism. These two approaches may provide more efficient and more correct results for structure-based molecular modeling for both purposes. The full agonist must have the hydrogen bond binding to Helix12 His421, is also an important judgment for virtual $\operatorname{LXR} \alpha$ modulators development. We conclude that computationally assessed qualities of crystal pockets are an important checkpoint that can help discover structurally novel nuclear receptor modulators and cost less.

\section{Summary points}

In silico approaches of molecular docking screening

- We can see that better crystal protein resolution did not ensure lower root-mean-square-deviation values after self-docking.

- 3IPU ligand-binding site was considered to be the best $x$-ray binding site for the new molecular docking modulator screening of liver $X$ receptor alpha $(L X R \alpha)$.

In vitro reporter gene assay

- In in vitro reporter gene assay, the 20 agonists or antagonists were confirmed.

- Four agonists $(\mathbf{1}, \mathbf{9}, 11$ and 22) had LXR $\alpha$ transactivation higher than or similar to the transactivation of L783483.

- Two antagonists ( 2 and 5 ) showed significant antagonism less than $20 \%$ more than control.

Distinguishing agonism

- To allow the maximum docking poses, we took the largest binding pocket, which may accommodate the greatest variety of $L X R \alpha$ ligand structures.

- 3FC6 has the largest-size ligand-binding pocket, might be a fit model to distinguish agonist and antagonist.

- We can safely draw a qualitative conclusion that the agonist must have the hydrogen bond binding to Helix12 His42.

- The main structural features for the $\operatorname{LXR} \alpha$ agonists are having strong hydrogen acceptors on the heads of agonists.

\section{Supplementary data}

To view the supplementary data that accompany this paper please visit the journal website at: www.future-science.com/doi/suppl /10.4155/fmc-2020-0069

\section{Financial \& competing interests disclosure}

We are grateful to the Ministry of Science and Technology of Taiwan for financial support (grant no. MOST107-2119-M-037-001MY2). This study is also supported partially by Kaohsiung Medical University Research Center (grant no. KMU-TC108A03-4). Huang received a grant from the I-Shou University (grant no. ISU101-04-01). The authors have no other relevant affiliations or financial involvement with any organization or entity with a financial interest in or financial conflict with the subject matter or materials discussed in the manuscript apart from those disclosed.

No writing assistance was utilized in the production of this manuscript. 


\section{References}

Papers of special note have been highlighted as: $\bullet$ of interest; $\bullet \bullet$ of considerable interest

1. Zheng J, Li Z, Manabe Y et al. Siphonaxanthin, a carotenoid from green algae, inhibits lipogenesis in hepatocytes via the suppression of liver X receptor $\alpha$ activity. Lipids 53(1), 41-52 (2018).

2. Zhou Y, Yu S, Cai C, Zhong L, Yu H, Shen W. LXRa participates in the mTOR/S6K1/SREBP-1c signaling pathway during sodium palmitate-induced lipogenesis in HepG2 cells. Nutr. Metab. 15, 31 (2018).

3. Ji L, Zhang B, Zhao G. Liver X receptor alpha ( $L X R \alpha)$ promoted invasion and EMT of gastric cancer cells by regulation of NF- $\mathrm{B}$ activity. Hum. Cell 30(2), 124-132 (2017).

4. Ju X, Huang P, Chen M, Wang Q. Liver X receptors as potential targets for cancer therapeutics. Oncol. Lett. 14(6), 7676-7680 (2017).

5. Ma Z, Deng C, Hu W et al. Liver X receptors and their agonists: targeting for cholesterol homeostasis and cardiovascular diseases. Curr. Issues Mol. Biol. 22, 41-64 (2017).

6. El-Gendy BEM, Goher SS, Hegazy LS, Arief MMH, Burris TP. Recent advances in the medicinal chemistry of liver x receptors. J. Med. Chem. 61(24), 10935-10956 (2018).

7. Everett JR. Academic drug discovery: current status and prospects. Expert Opin. Drug Discov. 10(9), 937-944 (2015).

8. Lin YT, Huang KJ, Tseng CK, Chen KJ, Wang HM, Lee JC. Efficient in silico assay of inhibitors of hepatitis C virus RNA-dependent RNA polymerase by structure-based virtual screening and in vitro evaluation. Assay Drug Dev. Technol. 9(3), 290-298 (2011).

9. Lee JH, Lin WC, Wen TK, Wang C, Lin YT. Inhibiting two cellular mutant epidermal growth factor receptor tyrosine kinases by addressing computationally assessed crystal ligand pockets. Future Med. Chem. 11(8), 833-846 (2019).

-. Previous description for our self-docking procedure.

10. Pitera JW. Expected distributions of root-mean-square positional deviations in proteins. J. Phys. Chem. B 118(24), 6526-6530 (2014).

11. Diamond IR, Grant RC, Feldman BM et al. Defining consensus: a systematic review recommends methodologic criteria for reporting of Delphi studies. J. Clin. Epidemiol. 67(4), 401-409 (2014).

12. Lipinski CA, Lombardo F, Dominy BW, Feeney PJ. Experimental and computational approaches to estimate solubility and permeability in drug discovery and development settings. Adv. Drug Deliv. Rev. 46(1-3), 3-26 (2001).

13. Yun C, Dasgupta R. Luciferase reporter assay in Drosophila and mammalian tissue culture cells. Curr. Protoc. Chem. Biol. 6(1), 7-23 (2014).

14. Svensson S, Ostberg T, Jacobsson M et al. Crystal structure of the heterodimeric complex of LXRalpha and RXRbeta ligand-binding domains in a fully agonistic conformation. EMBO J. 22(18), 4625-4633 (2003).

15. Prufer K, Hernandez C, Gilbreath M. Mutations in the AF-2 region abolish ligand-induced intranuclear immobilization of the liver $X$ receptor alpha. Exp. Cell Res. 314(14), 2652-2660 (2008).

16. Jin $Y$, Duan M, Wang X et al. Communication between the ligand-binding pocket and the activation function-2 domain of androgen receptor revealed by molecular dynamics simulations. J. Chem. Inf. Model. 59(2), 842-857 (2019).

17. Venkatachalam CM, Jiang X, Oldfield T, Waldman M. LigandFit: a novel method for the shape-directed rapid docking of ligands to protein active sites. J. Mol. Graph. Model. 21(4), 289-307 (2003).

18. Gehlhaar DK, Verkhivker GM, Rejto PA et al. Molecular recognition of the inhibitor AG-1343 by HIV-1 protease: conformationally flexible docking by evolutionary programming. Chem. Biol. 2(5), 317-324 (1995).

19. Mayo SL, Olafson BD, Goddard WA. DREIDING: a generic force field for molecular simulations. J. Phys. Chem. 94(26), 8897-8909 (1990).

20. Maple JR, Dinur U, Hagler AT. Derivation of force fields for molecular mechanics and dynamics from ab initio energy surfaces. Proc. Natl Acad. Sci. USA 85(15), 5350-5354 (1988).

21. Brooks BR, Bruccoleri RE, Olafson BD, States DJ, Swaminathan S, Karplus M. CHARMM: a program for macromolecular energy, minimization and dynamics calculations. J. Comput. Chem. 4(2), 187-217 (1983).

22. Fogel GB, Corne DW, Pan Y. Computational Intelligence in Bioinformatics. John Wiley \& Sons, NJ, USA (2007).

23. Cole JC, Murray CW, Nissink JW, Taylor RD, Taylor R. Comparing protein-ligand docking programs is difficult. Proteins 60(3), 325-332 (2005).

-. Important ideas about root-mean-square deviation.

24. Jaye MC, Krawiec JA, Campobasso $\mathrm{N}$ et al. Discovery of substituted maleimides as liver X receptor agonists and determination of a ligand-bound crystal structure. J. Med. Chem. 48(17), 5419-5422 (2005).

25. Chao EY, Caravella JA, Watson MA et al. Structure-guided design of $N$-phenyl tertiary amines as transrepression-selective liver $\mathrm{X}$ receptor modulators with anti-inflammatory activity. J. Med. Chem. 51(18), 5758-5765 (2008).

26. Washburn DG, Hoang TH, Campobasso $\mathrm{N}$ et al. Synthesis and SAR of potent LXR agonists containing an indole pharmacophore. Bioorg. Med. Chem. Lett. 19(4), 1097-1100 (2009).

- Binding pocket on the crystal 3FC6 was used here to tell if a ligand is an agonist. 
27. Fradera X, Vu D, Nimz O et al. X-ray structures of the LXRalpha LBD in its homodimeric form and implications for heterodimer signaling. J. Mol. Biol. 399(1), 120-132 (2010).

- The binding pocket on the crystal 3IPU was used here in the virtual screening of molecular docking for LXR $\alpha$ modulators.

28. Matsui Y, Yamaguchi T, Yamazaki T et al. Discovery and structure-guided optimization of tert-butyl 6-(phenoxymethyl)-3-(trifluoromethyl)benzoates as liver X receptor agonists. Bioorg. Med. Chem. Lett. 25(18), 3914-3920 (2015).

29. Stachel SJ, Zerbinatti C, Rudd MT et al. Identification and in vivo evaluation of liver $\mathrm{x}$ receptor beta-selective agonists for the potential treatment of Alzheimer's disease. J. Med. Chem. 59(7), 3489-3498 (2016).

30. Berman HM, Westbrook J, Feng Z et al. The protein data bank. Nucleic Acids Res. 28(1), 235-242 (2000).

31. Muegge I, Martin YC. A general and fast scoring function for protein-ligand interactions: a simplified potential approach. J. Med. Chem. 42(5), 791-804 (1999).

32. Muegge I. PMF scoring revisited. J. Med. Chem. 49(20), 5895-5902 (2006).

33. Böhm HJ. On the use of LUDI to search the fine chemicals directory for ligands of proteins of known three-dimensional structure. J. Comput. Aided Mol. Des. 8(5), 623-632 (1994).

34. Böhm HJ. The development of a simple empirical scoring function to estimate the binding constant for a protein-ligand complex of known three-dimensional structure. J. Comput. Aided Mol. Des. 8(3), 243-256 (1994).

35. Böhm HJ. Prediction of binding constants of protein ligands: a fast method for the prioritization of hits obtained from de novo design or 3D database search programs. J. Comput. Aided Mol. Des. 12(4), 309-323 (1998).

36. Parrill A. Rational Drug Design: Novel Methodology and Practical Applications American Chemical Society, Washington DC, USA. (1999).

37. Jain AN. Scoring noncovalent protein-ligand interactions: a continuous differentiable function tuned to compute binding affinities. J. Comput. Aided Mol. Des. 10(5), 427-440 (1996).

38. Veber DF, Johnson SR, Cheng HY, Smith BR, Ward KW, Kopple KD. Molecular properties that influence the oral bioavailability of drug candidates. J. Med. Chem. 45(12), 2615-2623 (2002).

39. Lin YT, Chen GY. A scaffold-independent subcellular event-based analysis: characterization of significant structural modifications. $J$. Chem. Inf. Model. 52(2), 506-514 (2012).

40. Hoyt CT, Konotopez A, Ebeling C, Wren J. PyBEL: a computational framework for biological expression language. Bioinformatics 34(4), 703-704 (2018).

41. O’Boyle NM, Morley C, Hutchison GR. Pybel: a Python wrapper for the OpenBabel cheminformatics toolkit. Chem. Cent. J. 2, 5 (2008).

42. Halgren AT. Merck molecular force field. I. Basis, form, scope, parameterization and performance of MMFF94. J. Comput. Chem. 17(5 \& 6), 490-519 (1995).

43. Hoffer L, Chira C, Marcou G, Varnek A, Horvath D. S4MPLE-sampler for multiple protein-ligand entities: methodology and rigid-site docking benchmarking. Molecules 20(5), 8997-9028 (2015).

44. Ashtawy HM, Mahapatra NR. Boosted neural networks scoring functions for accurate ligand docking and ranking. J.Bioinform. Comput. Biol. 16(2), 1850004 (2018).

45. Charifson PS, Corkery JJ, Murcko MA, Walters WP. Consensus scoring: a method for obtaining improved hit rates from docking databases of three-dimensional structures into proteins. J. Med. Chem. 42(25), 5100-5109 (1999).

46. Wang R, Wang S. How does consensus scoring work for virtual library screening? An idealized computer experiment. J. Chem. Inf. Comput. Sci. 41(5), 1422-1426 (2001).

47. Clark RD, Strizhev A, Leonard JM, Blake JF, Matthew JB. Consensus scoring for ligand/protein interactions. J. Mol. Graph Model. 20(4), 281-295 (2002).

48. Bajorath J. Computational scaffold hopping: cornerstone for the future of drug design? 9(7), 629-631 (2017).

49. Dunlop TW, Vaisanen S, Frank C, Molnar F, Sinkkonen L, Carlberg C. The human peroxisome proliferator-activated receptor delta gene is a primary target of 1alpha,25-dihydroxyvitamin D3 and its nuclear receptor. J. Mol. Biol. 349(2), 248-260 (2005). 